\title{
$p$-Phenylenediamine sensitization is more prevalent in central and southern European patch test centres than in Scandinavian: results from a multicentre study
}

Jacob Pontoppidan Thyssen $^{1}$, Klaus Ejner Andersen ${ }^{2}$, Magnus Bruze $^{3}$, Thomas Diepgen ${ }^{4}$, Ana M. Giménez-Arnau $^{5}$, Margarida Gonçalo ${ }^{6}$, An Goossens $^{7}$, Christophe Le Coz $^{8}$, John McFadden ${ }^{9}$, Thomas Rustemeyer ${ }^{10}$, Ian R White ${ }^{9}$, Jonathan M. White ${ }^{9}$ and Jeanne Duus Johansen ${ }^{1}$

${ }^{1}$ National Allergy Research Centre, Department of Dermato-Allergology, Gentofte Hospital, University of Copenhagen, DK-2900 Hellerup, and ${ }^{2}$ Department of Dermatology, Odense University Hospital, University of Southern Denmark, DK-5000 Odense, Denmark, ${ }^{3}$ Department of Occupational and Environmental Dermatology, Malmö University Hospital, Lund University, S-20502 Malmö, Sweden, ${ }^{4}$ Department of Social Medicine, University Hospital Heidelberg, 69115 Heidelberg, Germany, ${ }^{5}$ Department of Dermatology, Hospital del Mar, IMAS, Universitat Autònoma de Barcelona, Barcelona, Spain, ${ }^{6}$ Department of Dermatology, University Hospital, P-3000-175 Coimbra, Portugal, ${ }^{7}$ Department of Dermatology, University Hospital, K. U. Leuven, 3000 Leuven, Belgium, ${ }^{8}$ Cabinet de Dermatologie, Strasbourg, France, ${ }^{9}$ Department of Cutaneous Allergy, St John's Institute of Dermatology, London SE1 7EH, UK, and ${ }^{10}$ Department of Dermatology, Free University Hospital, NL-1081 HV Amsterdam, The Netherlands

Background: Positive patch test reactions to $p$-phenylenediamine (PPD) are common. PPD is used in oxidative hair dyes and is also present in dark henna temporary 'tattoos'. Cross-sensitization to other contact allergens may occur. Because subjects sensitized to PPD are at risk of clinically severe reactions upon hair dyeing, there is a need for 'current' prevalence data on PPD sensitization.

Objectives: To compare PPD patch test results from dermatitis patients tested between 2003 and 2007 in 10 European patch test centres and to analyse the causes and determine relevance of positive PPD patch test reactions.

Materials: Patch testing was performed using PPD (1\% free base in petrolatum from Trolab (Almirall Hermal GmbH, Reinbeck, Germany) or Chemotechnique (Malmö, Sweden), equivalent to $0.090 \mathrm{mg} / \mathrm{cm}^{2}$ in the TRUE ${ }^{\mathbb{R}}$ test from MEKOS Laboratories AS). Statistical analysis was performed using the chi-squared test.

Results: The weighted average prevalence was 4.6\% among 21515 patients. PPD sensitization occurred more often in centres located in Central and Southern Europe than in Scandinavian centres (odds ratio $=2.40 ; 95 \%$ confidence interval $=2.07-2.78)$. The overall proportion of positive patch test reactions to PPD that were registered as being of either current or 'past' relevance was high (weighted average $53.6 \%$ and $20.3 \%$, respectively). Consumer hair dyeing was the most prominent cause of PPD sensitization (weighted average $41.8 \%$ ). Furthermore, occupational hair dye exposure $(10.6 \%)$ and cross-sensitization to textile dyes $(12.6 \%)$ were frequently reported.

Conclusions: PPD sensitization caused by exposure to hair dyes is frequent and remains a present problem for patients visiting contact dermatitis clinics, especially in patch test centres located in Central and Southern Europe.

Key words: allergy; cross-sensitization; hair dyes; $p$-phenylenediamine; relevance. (C) 2009 John Wiley \& Sons A/S.

Conflicts of interest: The authors have declared no conflicts. 
Positive patch test reactions to $p$-phenylenediamine (PPD) are frequent among patients with contact dermatitis in Europe $(1,2)$. The leading causes of sensitization are exposure to oxidative hair dyes and dark henna temporary 'tattoos', although patch test reactivity also may occur as a result of 'cross-sensitization' to other allergens. Because sensitized individuals are at risk of clinically severe reactions upon hair dyeing, there is a need for 'current' prevalence data on PPD sensitization as well as information about causative exposures. This study aimed to compare patch test results from patients with dermatitis tested between 2003 and 2007 in 10 European patch test centres and furthermore to compare their causes and determine relevance of positive PPD patch test reactions.

\section{Materials and Methods}

\section{Patch test}

Between 2003 and 2007, patch testing with PPD ( $1 \%$ free base in petrolatum from Trolab or Chemotechnique, equivalent to $0.090 \mathrm{mg} / \mathrm{cm}^{2}$ in the TRUE $^{\mathbb{R}}$ test from MEKOS Laboratories AS, Hillerød, Denmark) was performed according to the recommendation of the International Contact Dermatitis Research Group (3) in 10 European patch test centres (Amsterdam, Barcelona, Coimbra, Gentofte, Heidelberg, Leuven, London, Malmö, Odense, and Strasbourg). Patch test systems included the TRUE test (MEKOS Laboratories AS), Van-der-Bend ${ }^{\mathbb{B}}$ square chambers (Brielle, the Netherlands), and Finn Chambers ${ }^{\mathbb{R}}$ (Epitest Ltd Oy, Tuusula, Finland) (Table 1). Most centres performed early (D2, D3, and D4) as well as late (D7) patch test readings (Table 1). Relevance of positive patch test reactions to PPD was assessed by the consulting physician: 'current relevance' was registered when a patient presented with a dermatitis reaction in combination with a history of current exposure to a source of PPD, 'past relevance' was registered when a patient had a positive patch test reaction to PPD in combination with a medical history of a past dermatitis reaction caused by exposure to a source of PPD, and 'unknown relevance' or 'missing' was registered in PPD-sensitized dermatitis patients where no relation to current or past PPD exposure could be assessed or where no registrations had been performed by the physician. Relevant exposures ('hair dyes - consumer', 'hair dyes occupational', 'henna tattoos', 'textiles/shoes/ gloves', 'black rubber', 'sulfonamides', 'benzocaine', and 'other or unknown') were based on patient interviews.

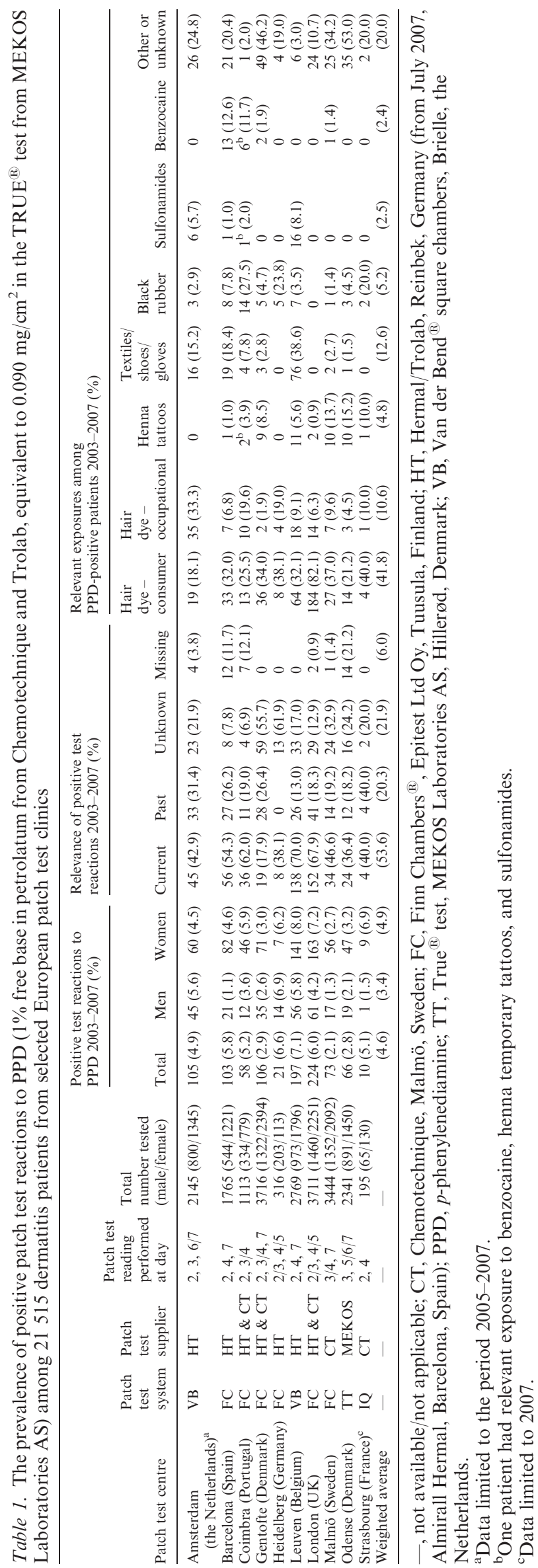




\section{Statistical analysis}

PPD patch test results from centres located in Central and Southern Europe (i.e. countries located south of Denmark and Sweden) were compared with results from centres in Scandinavia using the chi-squared test. Associations were expressed as odds ratios (ORs) with confidence intervals (CIs) of $95 \%$. Statistical significance was considered to be present if the $P$ value was below 0.05 . Furthermore, prevalence estimates were ranked and the median selected. Also, a weighted average prevalence was conducted $\left[\left(n_{1} \times \%_{1}+n_{2} \times \%_{2}+n_{\mathrm{n}} \times \%_{\mathrm{n}}\right) /\left(n_{1}\right.\right.$ $\left.+n_{2}+n_{\mathrm{n}}\right)$ ]. Data analysis was performed using Microsoft Excel 2002 (Microsoft, Redmond, Seattle, WA, USA).

\section{Results}

According to the MOAHLFA index, patch test populations from the 10 centres were generally comparable (Table 2). The Heidelberg clinic had the largest proportion of male patients $(60.4 \%)$, patients with hand dermatitis $(53.5 \%)$, and patients with occupational dermatitis $(61.1 \%)$. Also, the Malmö clinic reported a high proportion of patients with hand dermatitis $(48.6 \%)$ and occupational dermatitis $(47.9 \%)$. The prevalence of hand dermatitis $(15 \%)$ and facial dermatitis $(6.7 \%)$ was the lowest in Barcelona and Heidelberg, respectively.

Table 1 shows the prevalences of PPD sensitization among a total of 21515 patients with dermatitis (7944 men and 13571 women) tested between 2003 and 2007. The median prevalence was $5.1 \%$, and the weighted average was $4.6 \%$. The highest prevalence was identified in Leuven $(7.1 \%)$ and the lowest in Malmö $(2.1 \%)$. Fig. 1 shows the development of contact sensitization between 2003 and 2007. Patch test results from Heidelberg were not included in the figure as the proportion of occupational dermatitis was very high (Table 1). Furthermore, results from Strasbourg were not included as only 1 year (2007) was reported. The prevalences of PPD sensitization were generally higher in central and southerly located centres (i.e. Amsterdam, Barcelona, Coimbra, Heidelberg, Leuven, London, and Strasbourg) than in Scandinavian centres (i.e. Odense, Gentofte, and Malmö). This difference was statistically significant $(\mathrm{OR}=2.40, \mathrm{CI}=$ 2.07-2.78). The prevalence of PPD sensitization was generally higher among women than among men except in Amsterdam and Heidelberg where men showed more PPD sensitization than women.

The overall proportion of positive patch test reactions to PPD that were registered as being of

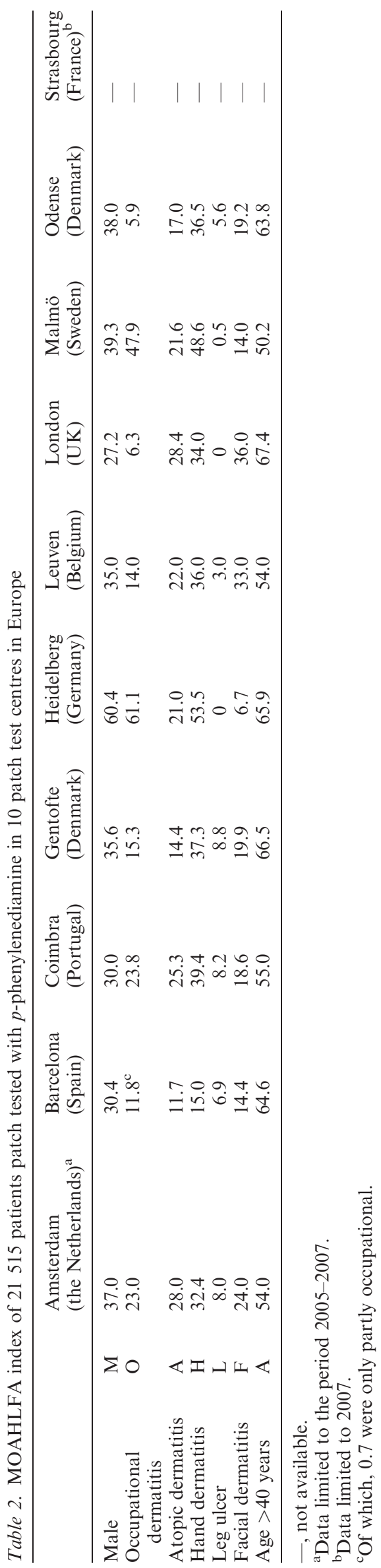




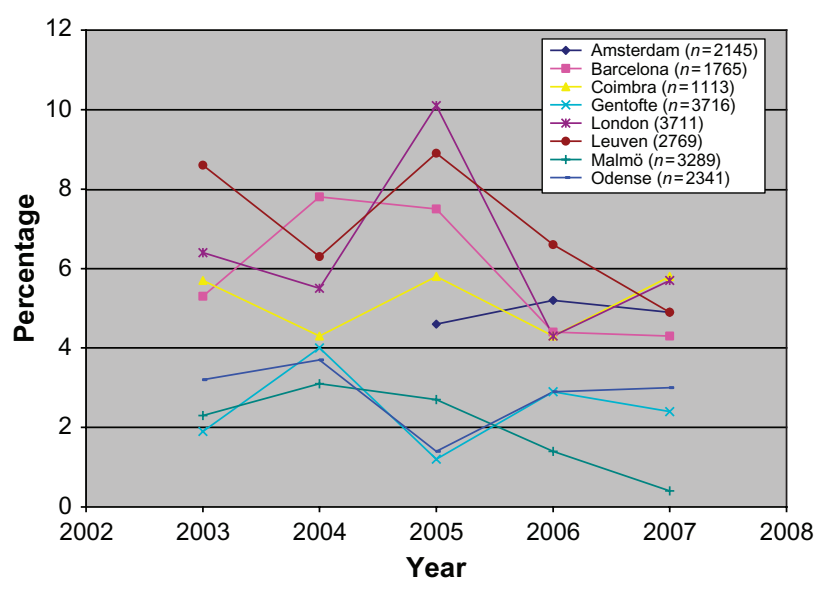

Fig. 1. The prevalence of positive patch test reactions to $p$-phenylenediamine $(1 \%$ free base in petrolatum from Chemotechnique and Trolab, equivalent to $0.090 \mathrm{mg} / \mathrm{cm}^{2}$ in the TRUE $^{\mathbb{R}}$ test from MEKOS Laboratories AS) among 21515 dermatitis patients from selected European patch test clinics.

either current or 'past' relevance was high (weighted average $53.6 \%$ and $20.3 \%$, respectively) (Table 1). Exposure to hair dyes (both occupational and consumer) was by far the most frequent cause of patch test reactivity to PPD. Hence, consumer hair dye exposure was registered in more than one-third of all patients (range 18.1-82.1\%, median prevalence $32.1 \%$, and weighted average $41.8 \%$ ), whereas occupational hair dye exposure was registered in around one of every 10 patients (range $4.5-33.3 \%$, median prevalence $9.1 \%$, and weighted average $10.6 \%$ ). Henna tattoos were less frequently reported in most centres (range 0 $15.2 \%$, median prevalence $3.9 \%$, and weighted average $4.8 \%$ ). Cross-sensitivity to chemically related compounds were reported following exposure to textiles, shoes, and gloves among $38.6 \%$ of PPD-positive patients in Leuven but only among $2.8 \%$ in Gentofte (range $0-38.6 \%$, median prevalence $2.7 \%$, and weighted average $12.6 \%$ ). Similarly, exposure to black rubber was reported among 27.5\% patients in Coimbra and among $23.8 \%$ in Heidelberg and to benzocaine among $12.6 \%$ in Barcelona and $11.7 \%$ in Coimbra.

\section{Discussion}

This study shows that PPD sensitization was significantly more prevalent among dermatitis patients tested between 2003 and 2007 in central and southerly located patch test centres (i.e. Amsterdam, Barcelona, Coimbra, Heidelberg, Leuven, London, and Strasbourg) than in Scandinavian centres (i.e. Malmö, Gentofte, and Odense) (Fig. 1 and Table 2). Because consumer and occupational hair dye exposure was frequently reported from all centres, the higher prevalence of PPD sensitization in central and southerly located centres is probably explained by the use of darker shades of hair dyes in these countries. Furthermore, individuals with darker hair may tend to dye their hair more often than those with blond hair as grey hair in dark-haired individuals is more easily spotted. As PPD-related substances are likely to be more common in hair dye products intended for dark hair than those intended for blond hair, a higher sensitization rate is to be expected in countries where the population has dark hair. Although Dutch people are often recognized for their blond coloration, Amsterdam also has a large ethnic minority. Thus, the high prevalence of PPD sensitization identified in Amsterdam may possibly be explained by immigrants from the former Dutch colonies. In general, centres with a large immigrant population from outside Europe may register higher prevalence rates (4). As this could potentially distort the results of our analysis, it should be noted that both Malmö and Copenhagen have large ethnic groups. Exposure to henna tattoos does not explain the difference between northerly and southerly located centres as henna temporary tattoos were infrequently reported from most centres except Gentofte (8.5\%), Malmö (13.7\%), and Odense (15.2\%) (Table 2). Similarly, recent data from St Johns Institute, London, showed that henna tattoo exposure only caused $2.6 \%$ of positive PPD patch test reactions (4). Furthermore, the higher prevalence of cross-sensitivity reported from, for example Amsterdam, Barcelona, Coimbra, and Leuven does not explain the higher prevalences of PPD sensitization in central and southerly located centres because Gentofte and Odense instead of cross-sensitivity may have reported other or unknown exposures. Thus, hair dye exposure (both among consumers as well as hairdressers and beauticians) remains the most important cause of PPD sensitization among European dermatitis patients and probably among subjects with dark hair. Of note, active sensitization from patch testing with PPD seems only to be of minor importance, if any, as the prevalence of PPD allergy was significantly different in the European centres. However, we have no data to establish the number of times patients have been patch tested.

The prevalence rates registered in this patientbased study were generally higher than those from patch studies performed in the general population $(5,6)$. Mirshahpanah and Maibach recently reviewed the literature and found that the average prevalence of PPD allergy in the general 
population was $1.5 \%$ (6). However, the high average prevalence was mainly explained by a significant contribution from a large Thai study including 2545 participants (the prevalence of PPD allergy was 2.3\%) (7), whereas a Danish patch test study with 469 participants found that only $0.2 \%$ were allergic to PPD (8). Of note, the patch test systems as well as the patch test reading schedules in these studies differed $(8,9)$. The clinical epidemiology and drug utilization research method (CEDUR) was used to estimate the 10 -year prevalence of PPD allergy in the general population in Denmark and Germany (9). The CEDUR results suggested that $0.4-0.5 \%$ of Danish adults and $0.8-1.8 \%$ of Germans (of all ages) had PPD allergy (9). Finally, Mirshahpanah and Maibach calculated a 'patch test positivity ratio' as the percentage of positive patch test reactions to PPD registered among dermatitis patients within the North American Contact Dermatitis Group (NACDG) and the European Environmental Contact Dermatitis Research Group (EECDRG), respectively, versus the prevalence registered among subjects in the general population (6). The analysis showed that the NACDG ratio was 3.2, whereas the EECDRG ratio was only 2.1. This finding suggests that PPD allergy is more prevalent among North American dermatitis patients than among European patients.

This study showed some variations regarding the frequency of different sources of PPD exposure (Table 1). Exposure to topical treatment modalities such as sulfonamides and benzocaine was frequently reported in Barcelona, Coimbra, Amsterdam, and Leuven but rarely in Scandinavian countries. The reason is that these substances are usually not used topically in Scandinavian countries. Furthermore, black rubber was frequently reported in Coimbra $(27.5 \%)$, which was explained by an unusual accumulation of industrial cases. Taken together, they confirm that positive patch test reactions to PPD are mainly caused by exposure to oxidative hair dyes and henna temporary tattoos (and cross-sensitivity to textile dyes in mainly Southern Europe), whereas exposures to substituted PPD in, for example printing ink, film developer, X-ray fluid, and rubber are generally infrequent causes. Furthermore, positive PPD patch test reactions may appear as a result of cross-sensitivity to chemically related compounds benzocaine (10), $N$-isopropyl- $N$-phenyl-para-phenylenediamine (11), $p$-toluenediamine (12), and sulfonamides. Of note, the overall proportion of positive reactions to PPD that were registered as being of current and past relevance was high as almost three of four reactions had relevance. The high propor- tion of current relevance was also in accordance with previous reports $(44-64 \%)(13,14)$. However, it should be remembered that positive PPD patch test reaction with 'unknown' or 'past' relevance is also of potential importance as these subjects are at risk of developing clinical reactions upon hair dyeing. Ho et al. recently identified a strong linear relationship between the strength of patch test reaction and the continuation with hair dyeing among patients in London (10). In conclusion, our data emphasize that PPD sensitization caused by exposure to hair dyes is frequent and, furthermore, that it remains a present problem for patients visiting contact dermatitis clinics, especially in central and southerly located European patch test centres.

\section{Acknowledgement}

The study was funded by Copenhagen County Research Foundation.

\section{References}

1. Thyssen J P, White J M L. Epidemiological data on consumer allergy to p-phenylenediamine. Contact Dermatitis 2008: 59: 327-343.

2. Thyssen J P, Johansen J D, Menné T. Contact allergy epidemics and their controls. Contact Dermatitis 2007: 56: 185-195.

3. Wilkinson D S, Fregert S, Magnusson B et al. Terminology of contact dermatitis. Acta Derm Venereol 1970: 50: 287-292.

4. Patel S, Basketter D A, Jefferies D et al. Patch test frequency to p-phenylenediamine: follow up over the last 6 years. Contact Dermatitis 2007: 56: 35-37.

5. Thyssen J P, Linneberg A, Menné T, Johansen J D. The epidemiology of contact allergy in the general populationprevalence and main findings. Contact Dermatitis 2007: 57: 287-299.

6. Mirshahpanah P, Maibach H I. Relationship of patch test positivity in a general versus an eczema population. Contact Dermatitis 2007: 56: 125-130.

7. White J M, Gilmour N J, Jeffries D et al. A general population from Thailand: incidence of common allergens with emphasis on para-phenylenediamine. Clin Exp Allergy 2007: 37: 1848-1853.

8. Nielsen N H, Linneberg A, Menné T et al. Allergic contact sensitization in an adult Danish population: two crosssectional surveys eight years apart (the Copenhagen Allergy Study). Acta Derm Venereol 2001: 81: 31-34.

9. Thyssen J P, Uter W, Schnuch A, Linneberg A, Johansen J D. 10-year prevalence of contact allergy in the general population in Denmark estimated through the CE-DUR method. Contact Dermatitis 2007: 57: 265-272.

10. Ho S G, Basketter D A, Jefferies D, Rycroft R J, White I R, McFadden J P. Analysis of para-phenylenediamine allergic patients in relation to strength of patch test reaction. $\mathrm{Br} J$ Dermatol 2005: 153: 364-367.

11. Uter W, Lessmann H, Geier J, Becker D, Fuchs T, Richter G. The spectrum of allergic (cross-)sensitivity in clinical patch testing with 'para amino' compounds. Allergy 2002: 57: 319-322. 
12. Xie Z, Hayakawa R, Sugiura M et al. Experimental study on skin sensitization potencies and cross-reactivities of hairdye-related chemicals in guinea pigs. Contact Dermatitis 2000: 42: 270-275.

13. Fregert S, Hjorth N, Magnusson B et al. Epidemiology of contact dermatitis. Trans St Johns Hosp Dermatol Soc 1969: 55: $17-35$.

14. Devos S A, Van D V. The risk of active sensitization to PPD. Contact Dermatitis 2001: 44: 273-275.
Address:

Jacob Pontoppidan Thyssen, MD

National Allergy Research Centre

Department of Dermatolo-Allergology Gentofte Hospital

University of Copenhagen

2900 Hellerup

Denmark

Tel: +45 39777307

Fax: +45 39777118

e-mail: jacpth01@geh.regionh.dk 